\title{
Downregulation of PLK1 by RNAi attenuates the tumorigenicity of esophageal squamous cell carcinoma cells via promoting apoptosis and inhibiting angiogenesis
}

\author{
C. L. ZHAO ${ }^{1}$, J. Y. JU' ${ }^{2}$, W. GAO ${ }^{2}$, W. J. YU ${ }^{1}$, Z. Q. GAO', W. T. LI ${ }^{2, *}$ \\ ${ }^{1}$ College of Pharmacy and Biological Science, ${ }^{2}$ College of Basic Medicine, Weifang Medical University, Weifang 261053, China \\ *Correspondence: liwentong11@163.com
}

Received December 23, 2014 / Accepted May 13, 2015

\begin{abstract}
Polo-like kinase 1(PLK1) is essential for the maintenance of genomic stability during mitosis. PLK1 has been reported to be upregulated in several solid tumors, including esophageal squamous cell carcinoma (ESCC). However, the role of PLK1 in tumorigenesis of ESCC remains undetermined. We used siRNA and lentivirus-mediated PLK1 RNA interference to investigate the tumor suppressor function of PLK1 reduction in ESCC cells. Flow cytometry and Terminal deoxynuleotidyl transferase-mediated nick-end labeling assay in vitro, as well as immunohistochemitry analysis of Caspase-3 and CD31 in s.c. tumor tissue section, were performed. Knock down of PLK1 expression significantly suppressed the ability of ESCC cells to form colonies in plastic and soft agar. PLK1 reduction mediated by lentivirus caused growth suppression of ESCC in nude mice. Caspase-3 upregulation further indicated that dysregulated apoptosis might contribute to reduced tumorigenecity. In particular, downregulation of CD31 suggested that PLK1 reduction-induced angiogenesis inhibition may also contribute, at least in part, to attenuated tumorigenecity. These findings indicate that PLK1 might play roles in tumorigenesis of ESCC and that PLK1 might be a potential gene therapy target in ESCC. Apoptosis induction together with decreased angiogenesis might be involved in the mechanism of tumor suppressor function of RNA interference targeting PLK1.
\end{abstract}

Key words: PLK1, ESCC, tumorigenecity, angiogenesis

Esophageal cancer is one of the most common cancer worldwide [1].Esophageal squamous cell carcinoma (ESCC), the major histologic form of esophageal cancer, dominates in most parts of the world, especially in China [2]. Multiple genetic changes have been found in ESCC, but little is known about major oncogenes and tumor suppressor genes involved in the tumorigenesis.

Human polo-like kinase 1 (PLK1) is a highly conserved serine/threonine kinase that regulates a multitude of mitotic processes [3]. PLK1 has been reported to be overexpressed in several solid tumors, such as esophageal, prostate, and colon cancer [4-9]. Depletion of PLK1 by siRNA dramatically inhibited some cancer cell growth and induced apoptosis [10-13]. But, so far, the precise role of PLK1 in ESCC tumorigenesis is largely unknown.

In the present study, we have elucidated the fundamental question of whether PLK1 dysregulation was involved in ESCC tumorigenesis. We have demonstrated that reduction of PLK1 by siRNA attenuated the tumorigenicity of ESCC cells TE-8, as illustrated by decreased colony formation capacity and anchorage-independent growth, and reduced tumor growth in nude mice. More interestingly, we found that knock down of PLK1 expression resulted in inhibition of angiogenesis in addition to apoptosis induction.

\section{Materials and methods}

Cell culture. Human ESCC cell lines Eca-109, TE-8, TE-10 and TE-15 were purchased from Shanghai Cell Bank, Chinese academy of sciences (Shanghai, China). Cells were cultured in RPMI 1640 medium supplemented with 10\% fetal bovine serum, penicillin $(100 \mathrm{U} / \mathrm{mL})$ and streptomycin $(100 \mathrm{mg} / \mathrm{mL})$ at $37^{\circ} \mathrm{C}$ humidified atmosphere of $5 \% \mathrm{CO} 2$.

Western blot analysis. The proteins were separated by SDS-PAGE and then transferred to polyvinylidene difluoride membranes (Millipore Bedford, MA). Blots were blocked and then probed with antibodies against PLK1 (1:1000 dilution, Santa Cruz, USA), and B-actin (1:5000 dilution, Santa Cruz, 
USA). After washing, the blots were incubated with horseradish peroxidase-conjugated secondary antibodies. The protein bands were visualized using super ECL detection reagent (Applygen, China).

Small interfering RNA synthesis and transfection. siRNA sequences targeting Human PLK1 were chemically synthesized for screening (GenePharma Co., China). The sequences of the siRNA for the PLK1 gene (PLK1-siRNA) were selected from nucleotide sequences 1418-1438 (siRNA-PLK1-1, sense sequence: 5'-GCGUGACGGCACUGAGUCCUATT-3', antisense sequence: 5'-UAGGACUCAGUGCCGUCACGCTT-3'); 183-203 (siRNA-PLK1-2, sense sequence: 5'- GGGCGGCUUUGCCAAGUGCUTT-3', antisense sequence: 5'- AGCACUUGGCAAAGCCGCCCTT-3'). A sequence non-specific to any known gene was used as a negative control (GenePharma Co., China). Transfection was performed in $80 \%$ confluent cells using Lipo-fectAMINE (Invitrogen, USA) according to the manufacture's protocol.

Colony formation assay. TE- 8 cells were treated with the siRNA for $48 \mathrm{~h}$ and then digested to a single cell suspension. A total of 200 cells were plated into $60 \mathrm{~mm}$ culture dishes and kept for 14 days, and the medium was changed once every week. The plates were taken out to observe the colony formation, fixed in methyl alcohol and stained with Giemsa. Cell clones over 50 cells were counted using a grid. Three independent experiments were performed.

Soft agar assay. Soft agar assay was carried out according to the procedures described previously [14]. Suspensions containing 500 cells were seeded into the top of the agar. After 3 weeks of incubation at $37^{\circ} \mathrm{C}, 5 \% \mathrm{CO} 2$, the number of colonies with a diameter $>500 \mu \mathrm{m}$ was counted. Counts were expressed as the number of colonies per plate on average from three independent experiments.

Apoptosis analysis by flow cytometry. The flow cytometry assay was performed by propidium iodide staining. TE- 8 cells were seeded at a density of $4 \times 10^{5}$ in $35 \mathrm{~mm}$ culture dishes. After $24 \mathrm{~h}$, the cells were incubated with siRNA-PLK1-2 for $48 \mathrm{~h}$. Cells and supernatants were collected together and centrifuged at $1000 \mathrm{rpm}$ for $5 \mathrm{~min}$. The pellets were washed twice with ice-cold phosphate-buffer saline (PBS) and fixed overnight at $4^{\circ} \mathrm{C}$ in $70 \%$ ethanol. After they were washed twice with PBS, cells were incubated with $5 \mu \mathrm{g} / \mathrm{mL}$ propidium iodide and 50 $\mu \mathrm{g} / \mathrm{mL}$ RNaseA in PBS for $1 \mathrm{~h}$ at room temperature. Samples were analyzed using a fluorescence-activated cell sorter (FACS) (Becton Dickinson, New York, USA).

Terminal deoxynuleotidyl transferase-mediated nickend labeling assay. TE- 8 cells were grown on coverslips in RPMI 1640 complete medium for $24 \mathrm{~h}$. After the cells were incubated with siRNA-PLK1-2 for $48 \mathrm{~h}$, the coverslips were rinsed twice with ice-cold PBS and fixed and permeabilized by immersion in cold methanol for $20 \mathrm{~min}$. Then, the coverslips were rinsed with PBS and analyzed by using Terminal deoxynuleotidyl transferase-mediated nick-end labeling (TUNEL) System (Promega, USA). The assay was performed according to the user manual and apoptotic cells appeared in green color with the FITC filter under a fluorescent microscope.

Construction of recombinant lentivirus vector and preparation of lentivirus stocks. Synthetic double-stranded oligonucleotides with the following sequences were inserted into the BamH I and EcoR I -excised lentivirus vector pGLV/H1/GFP+Puro, sense: 5' - GATCCGGGCGGCTTTGCCAAGTG CTTTCAA GAGAAGCACTTGGCAAAGCCGCCCTTTTTT-3', antisense: 5'- AATTCA AAAA AGGGCGGCTTT-

A

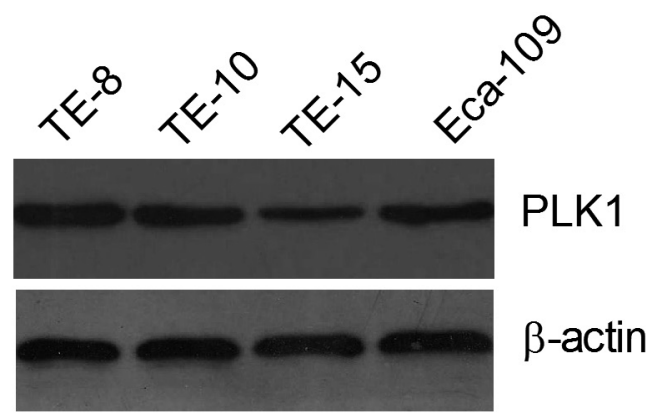

B
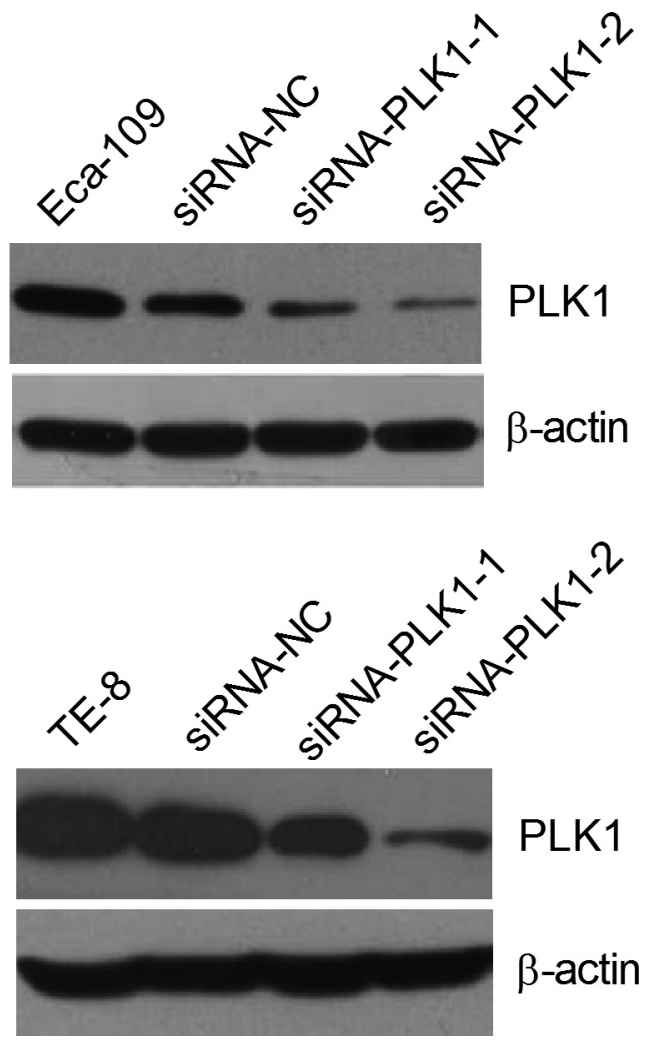

Figure 1. Reduction of PLK1 protein in ESCC by siRNA. (A) Western blot analysis of PLK1 expression in ESCC. (B) PLK1 protein level was examined in cells of Eca-109 and TE-8 transiently transfected with siRNAPLK1-1 or siRNA-PLK1-2. B-actin was used as an equal loading control for Western blot. 
GCCAAGTGCTTCTCTTGAA AGCA CTTGG CAAA GCCG CCC-3'. The recombinant lentivirus (LV-PLK1) was developed by the GenePharma Co., China. Briefly, The lentivirus (LV-PLK1) was cotransfected with Helper Vector into 293T cells by LipofectamineTM 2000. The lentivirus (LV-PLK1) was purified by cesium chloride gradient ultracentrifugation. Viral titer was determined using TCIG50 end-point dilution. The final titer of the purified viral vectors was $5.9 \times 10^{9} \mathrm{TU} / \mathrm{mL}$. The virus stocks were aliquoted and stored at $-80^{\circ} \mathrm{C}$.

Lentivirus-mediated gene therapy in nude mice. TE- 8 cells $\left(4 \times 10^{6}\right)$ in $0.1 \mathrm{~mL}$ of PBS were subcutaneously inoculated into the right flank of 4 -week-old $\mathrm{BALB} / \mathrm{c}$ nude mice. The experiment was divided into four groups with five mice in each group. When tumors reached the size of $50-100 \mathrm{~mm}^{3}$, $0.1 \mathrm{~mL}$ PBS, $0.1 \mathrm{~mL}$ LV-NC $\left(2 \times 10^{6} \mathrm{TU}\right), 0.1 \mathrm{~mL}$ LV-PLK1 $\left(2 \times 10^{6} \mathrm{TU}\right)$ was injected into tumor in each group, respectively. The injection was repeated once each three days. After 10 times of injection, tumors were excised under anesthesia, size-measured and weighted. Tumor volume was calculated by the formula $4 \pi \mathrm{r}_{1}{ }^{2} \mathrm{r}_{2} / 3(\mathrm{r} 1<\mathrm{r} 2)$.

Immunohistochemistry and microvessel quantitation. Immunohistochemistry was performed using the Biotin SP-
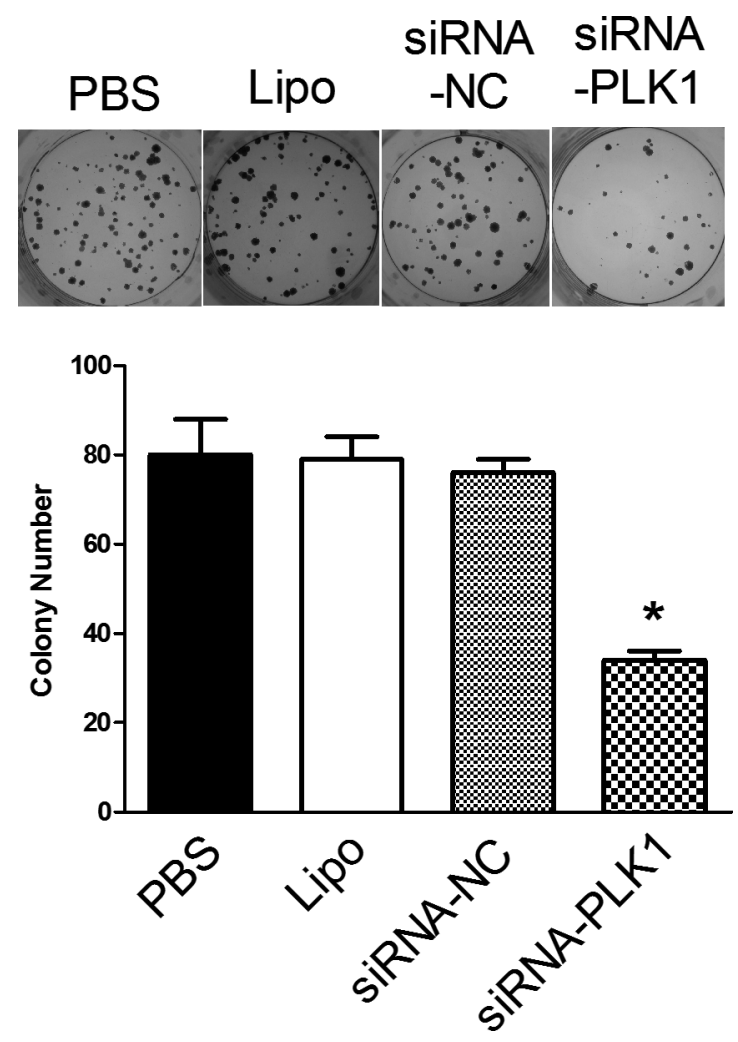

Figure 2. Effects of siRNA-PLK1 on colony formation. Cells were seeded in 6-well culture plate at 200 cells per well. Two weeks later, surviving colonies were stained and counted. Photographs of representatives plates are shown and histogram is the average value (mean $\pm S D)$ of 3 independent experiments. ${ }^{\star} \boldsymbol{P}<0.01$, compared to control cells.
HRP (DAB) Kit (Zhongshan biotech Co., China) following the manufacture's protocol. The anti-Caspase 3 and anti-CD31 (Sigma, USA) were used as the primary antibodies. Microvessel density was quantified by examining areas of vascular hotspots as previously described by Weidner et al [15]. Sections were scanned at low magnification $(\times 40)$ for the localization of vascular hotspots. The three most vascular areas of tumors were determined and then counted in the high power field $(\times 200)$. The values of the three sections were averaged, and the results were analyzed. Branching structures were counted as a single vessel as previously shown [16].

Statistical analysis. The data were analyzed by ANOVA. The statistical analysis was performed using SPSS 11.0 software and $P<0.05$ was considered significant.

\section{Results}

RNAi of PLK1 in ESCC cells. To investigate whether endogenous PLK1 is important in esophageal tumorigenesis, RNA interference (RNAi) was used to knock down PLK1 expression. TE-8 and Eca-109, which showed relatively higher levels of endogenous PLK1 expression (Fig. 1A), were treated with siRNAs targeting PLK1 (siRNA-PLK1-1 or siRNA-PLK1-2) or
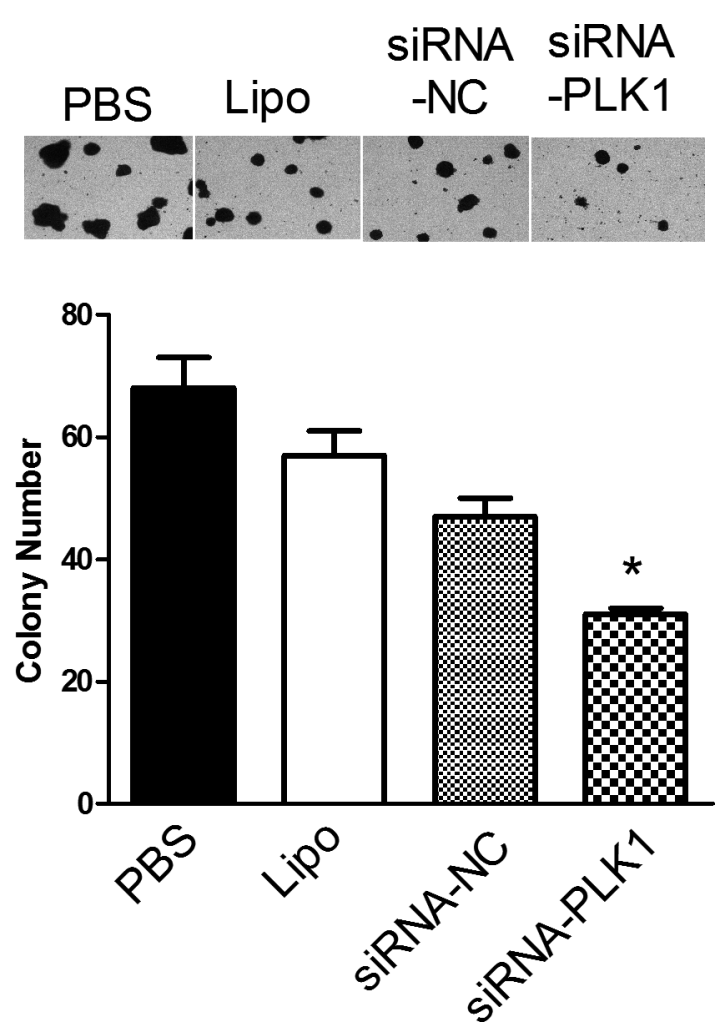

Figure 3. RNAi-mediated inhibition of PLK1 in TE-8-affected colony formation in soft agar. 500 cells were suspended in soft agar for assay of anchorindependent growth. After 3 weeks, colonies were counted. Representative photographs are shown and histogram is the average value (mean $\pm S D)$ of 3 independent experiments. ${ }^{\star} \boldsymbol{P}<0.01$, compared to control cells. 
siRNA-NC as a negative control. Compared with siRNA-NC and parent cells, treatment with specific siRNAs against PLK1 could dramatically reduce PLK1 expression in TE-8 and Eca109 cells at protein level. And siRNA-PLK1-2 worked more effectively (Fig. 1B). Thus, TE-8 and siRNA-PLK1-2 were selected for further analysis. Reduced expression of PLK1 suppressed TE-8 cell growth both in vitro and in vivo.

To elucidate the functional role of PLK1 in esophageal tumorigenesis, we first investigate the effect of silencing PLK1 expression on the malignant phenotype of ESCC TE-8 cells. Colony formation assay in monolayer culture showed that the number of surviving colonies of siRNA-PLK1 was markedly decreased compared to those of control cells, indicating that endogenous PLK1 expression is indispensible to the growth of TE-8 cells (Fig. 2). The anchorage-independent growth of TE-8 cells was also inhibited when TE-8 cells treated with siRNA-PLK1 were cultured in soft agar (Fig. 3). These findings indicate that silencing expression of PLK1 can suppress the malignant proliferation of TE- 8 cells.

To further evaluate the tumor suppression function of reduced PLK1 expression, experimental tumor gene therapy with the recombinant lentivirus (LV-PLK1) was carried out.
A

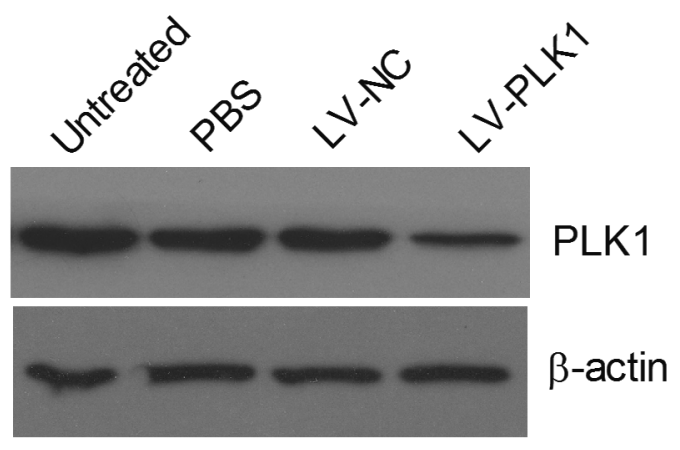

C

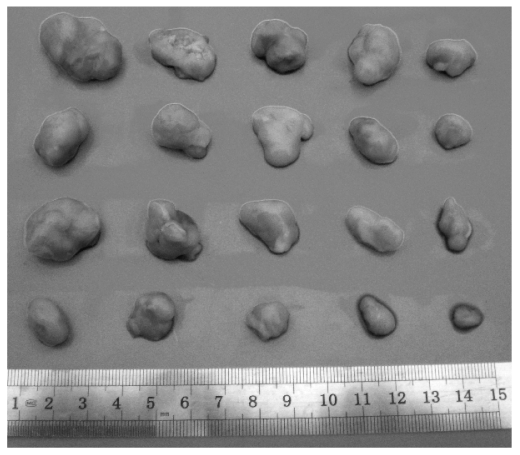

Untreated

PBS

LV-NC

LV-PLK1

D

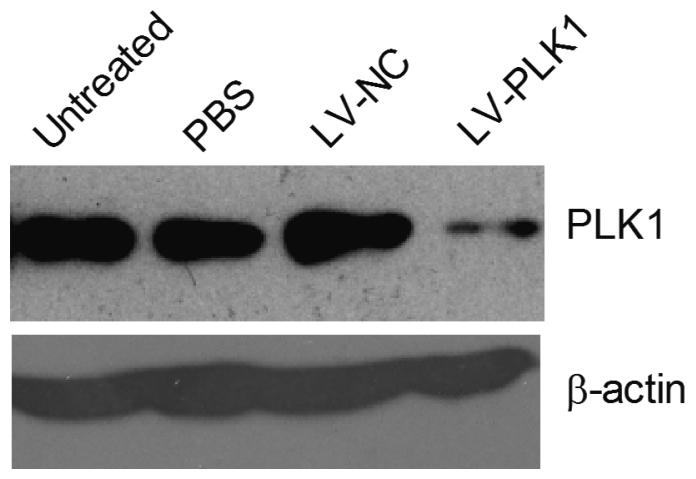

B
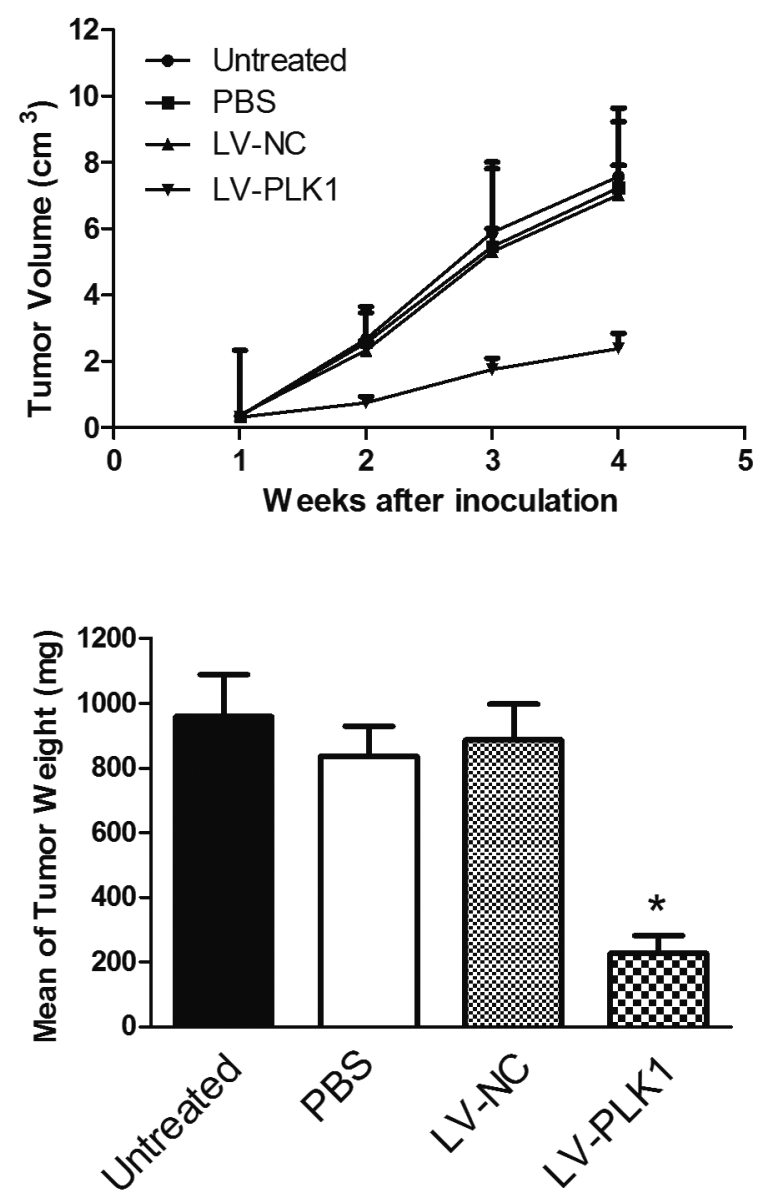

Figure 4. Growth suppression of TE-8 tumors by intratumor recombinant lentivirus (LV-PLK1) injection. (A) PLK1 reduction mediated by the recombinant lentivirus in TE-8 cells. (B) Tumor growth curve of s.c.tumors. (C) Tumor weight of different groups of nude mice. Values represent the mean for each group animals and plotted on a bar chart. ${ }^{*}, P<0.01$ versus controls. (D) Western blot analysis of PLK1in s.c.tumors tissues. 
The recombinant lentivirus could effectively inhibit PLK1 expression in TE- 8 cells (Fig. 4A). When tumors from inoculated TE- 8 cells in nude mice reached the size of $50-100 \mathrm{~mm}^{3}$, LV-PLK1, LV-NC, or PBS was injected into tumors. All mice injected with LV-PLK1 showed strong inhibition of tumor growth (Fig. 4B). The tumor volumes of the mice at Week 4 were decreased significantly (up to $63 \%$ ) in the mice with LV-PLK1 treatment compare to those of controls $(P<0.001)$ (Fig. 4C). The reduced expression level of PLK1 were further demonstrated in the xenograft tumor tissue injected LV-PLK1 by Western blot analysis (Fig. 4D).
Silencing PLK1 expression induced apoptosis of TE-8 cells. In view that tumor growth is determined by the balance of cell proliferation and programmed cell death, cell proliferation-related protein Ki-67 was analyzed on tumor sections by immunohistochemistry. No significant difference was observed between the LV-PLK1 treatment tumors and controls (data not shown). Then apoptosis was first examined in vitro by propidium iodide staining using flow cytometry. As shown in Fig. $5 \mathrm{~A}$, knock down of PLK1 by RNA interference resulted in the sub-G1 peak representing the apoptotic cell population. TUNEL assay was further used to support this finding.

\section{A}
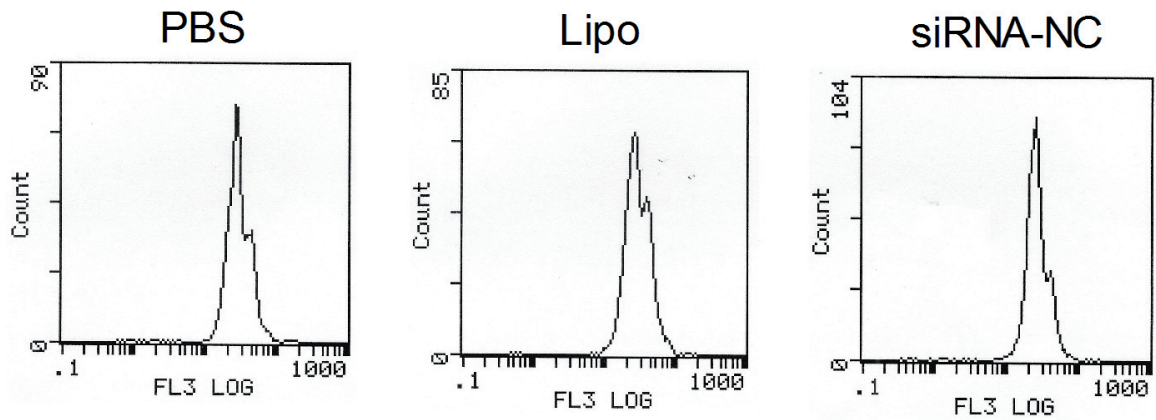

SIRNA-PLK1

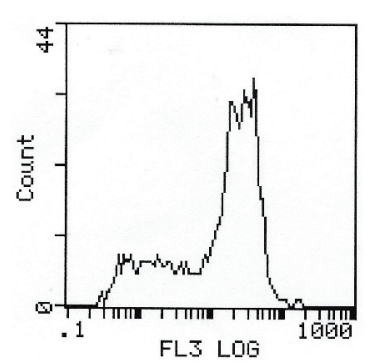

B

PBS

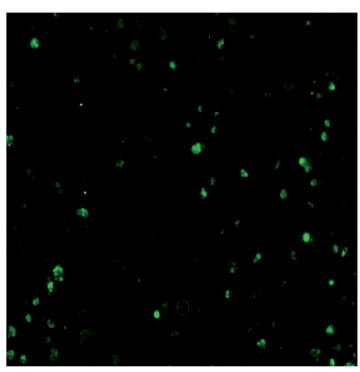

\section{Lipo}

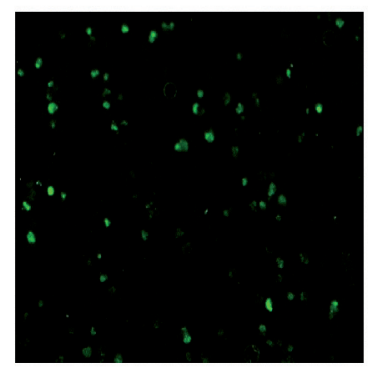

SiRNA-NC

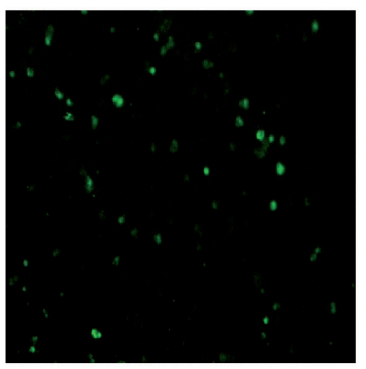

SiRNA-PLK1

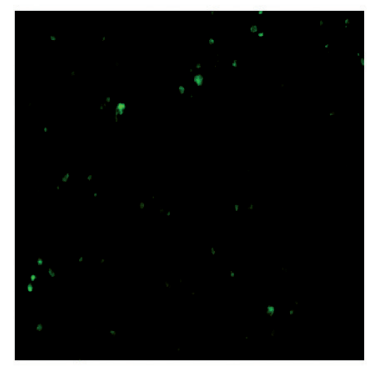

C

Untreated

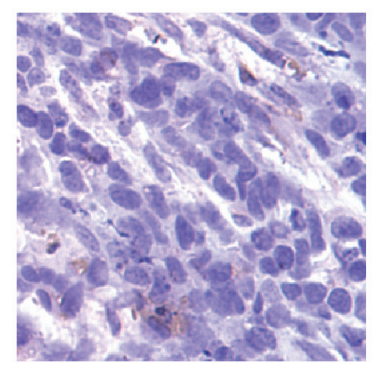

PBS

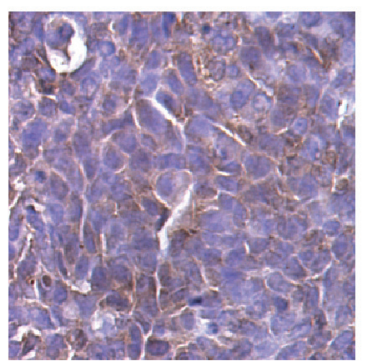

LV-NC

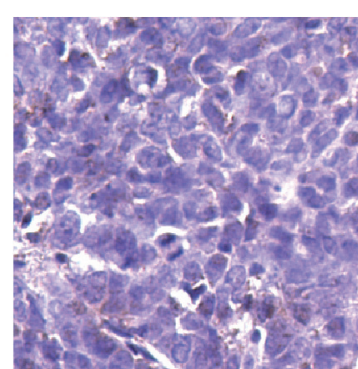

LV-PLK1

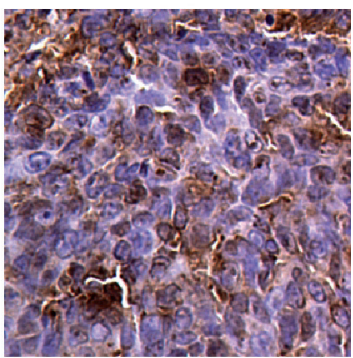

Figure 5. RNAi directed against PLK1 significantly promotes TE-8 cells apoptosis in vitro and in vivo. (A) Induction of apoptosis in TE-8 cells by PLK1siRNA treatment $(100 \mathrm{nmol} / \mathrm{L})$. Cells were labeled with PI and identified using flow cytometry. (B) TUNEL staining of apoptotic cells by siRNA treatment. Magnification: $\times 200$. (C) Immunohistochemitry staining of s.c.tumor tissue sections by cleaved Caspase 3 antibody. Magnification: $\times 200$. 
Very few apoptotic cells were found in TE- 8 cells treated with siRNA-NC, PBS and parental TE-8 cells. In contrast, more apoptotic cells were significantly detected in TE- 8 treated with siRNA-PLK1 (Fig. 5B). Next, we investigated the activation of the downstream effector Caspase such as Caspase-3. Increased expression of the active form of Caspase 3 expression were also detected in the tumor tissues of reduced PLK1 expression by immunhistochemistry staining using cleaved Caspase 3 antibody (Fig. 5C). These findings indicate that apoptosis mechanism is responsible for the decreased tumorigenecity derived from LV-PLK1.

Silencing PLK1 expression inhibited tumor angiogenesis. Xenograft tissue sections were also examined for CD31 expression by immunohistochemistry staining. CD31 is an indicator which is widely used for measure of angiogenesis. The tumors injected with LV-PLK1 showed a dramatic reduction of CD31 staining (Fig. 6A). As shown in Fig. 6B, microvessel density was significantly decreased in the tumors from LV-PLK1 treatment compared with the tumors from LV-NC, PBS and the parental cells $(\mathrm{P}<0.05)$. Western blot analysis further verified that reduced PLK1 expression and CD31 downregulation were detected in the tested samples from LV-PLK1 injected mice (Fig. 6C), suggesting that the attenuated tumorigenesis of TE-8 in nude mice by silencing expression of PLK1 was partly due to angiogenesis inhibition.

\section{Discussion}

The development and progression of ESCC have found to mainly arise from changes in some key genes that are associated with cell proliferation, apoptosis and genomic stability. Alterations in several oncogenes and tumor suppressor genes

A
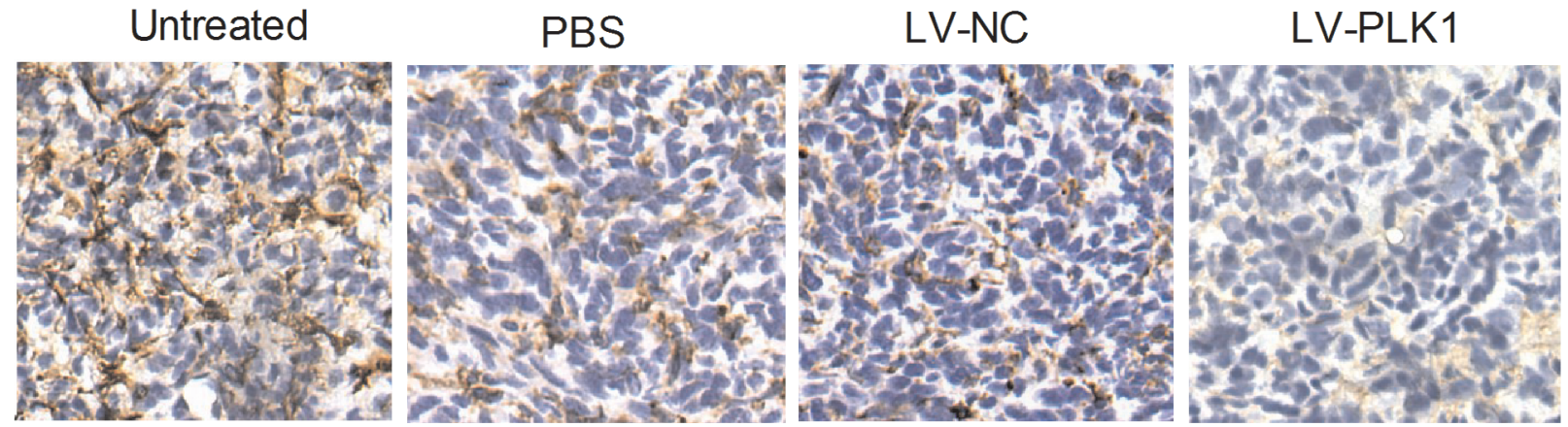

B

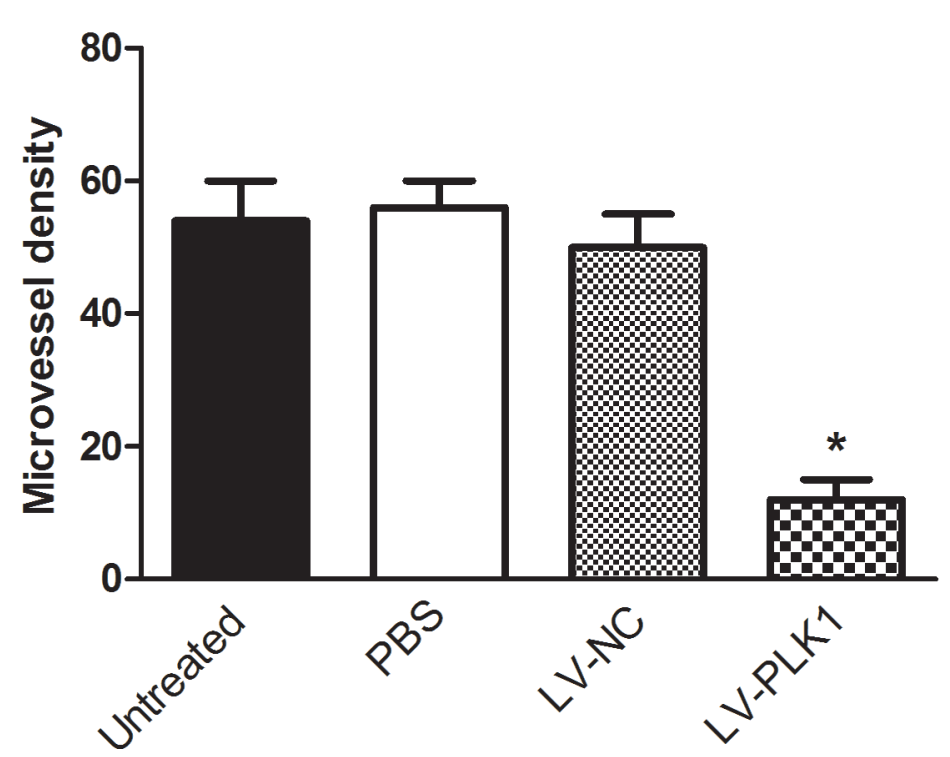

C
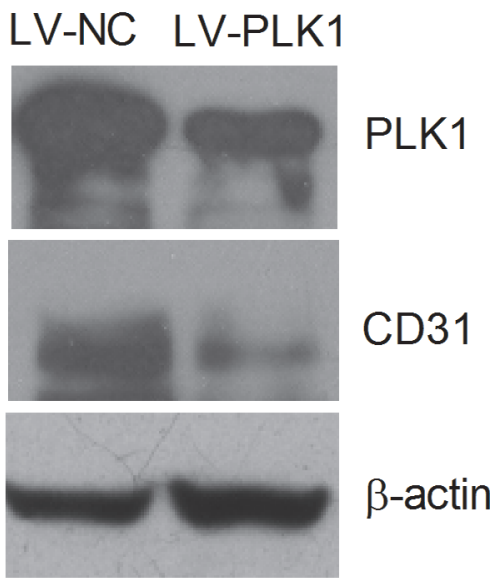

Figure 6. Silencing PLK1 expression inhibited angiogenesis of tumors from TE-8 cells. (A) Immunohistochemical analysis of tumor tissue sections stained with anti-human Caspase 3 antibody. Magnification: $\times 200$. (B) Microvessel density analysis in the tumor sections from TE-8 cells was done according to Materials and Methods. Column, mean; bars,SD. ${ }^{*}, P<0.01$. (C) PLK1 and CD31 expression of tumor tissues detected by Western blot. 
have been found such as p53, Rb, cyclin D1, and Aurora-A [17-22].

PLK1, is one of the most important regulators for the mitotic progression in mammalian cells. Moreover, overexpression of PLK1 is positively correlated with a broad range of human tumors. Previous studies have suggested that alteration of PLK1 expression level is a critical event in the development of human ESCC $[4,23,24]$. Thus, dysregulation of PLK1 gene might contribute to the malignant phenotype of ESCC cells. To determine this possibility, we investigated the effects of knockdown of PLK1 expression on the ESCC cells. In this study, we have demonstrated that the reduction of PLK1 by RNAi had a remarkable effect on malignant growth of ESCC cells in vitro and in vivo, implying that aberrant expression of PLK1 is not a consequence of carcinogenesis, but involved in malignant transformation of esophageal cells.

The existence of obvious apoptosis in vitro and in s.c. tumor tissue from nude mice inoculated with lentivirus-mediated PLK1 siRNA indicated that the suppressed malignant proliferation caused by the reduction of PLK1 expression was related with apoptosis. Our findings in vitro are consistent with the previous studies that silencing of PLK1 via siRNA causes induction of apoptosis in cancers including human esophageal cancer cells $[10,11,24,25]$. Furthermore, Feng et al [24] also revealed that downregulation of PLK1 resulted in a sharp decrease in pro-caspase- 3 and the cleavage of PARP in vitro. Our results in vivo demonstrated that the activation of Caspase 3 played a key role in ESCC cells apoptosis induced by PLK1 reduction. On the basis of these data, we suppose that the activation of Caspase 3 is one of the essential mechanisms for PLK1 reduction induced apoptosis. However, the detailed molecular mechanism through which PLK1 affects Caspase 3 remains to be clarified in further studies.

In addition, our study provides the first evidence of correlation between expression of PLK1 and angiogenesis. Immunohistochemical analysis with CD31 antibody, an indicator, which is widely used for measure of angiogenesis, that inhibition of tumor angiogenesis in ESCC could be induced by reduction of PLK1 expression. Studies have showed that angiogenesis is an essential factor in the growth and metastasis of many tumors including ESCC [26-28]. Although we did not observe the metastasis to other tissues from the lentivirus-mediated gene therapy in nude mice tumorigenecity assay for a short time, inhibition of tumor angiogenesis induced by PLK1 reduction must contribute in part to inhibit ESCC cell growth. Further studies should be addressed to elucidate the molecular mechanism of PLK1 in ESCC angiogenesis.

In conclusion, reduction of PLK1 expression mediated by RNAi suppressed the malignant phenotype of ESCC cell in vitro and in vivo. Especially, our results indicated that PLK1 reduction exerted the antitumor activity in ESCC cell growth at least partly mediated by inhibition of cell proliferation and tumor angiogenesis, as well as apoptosis induction. Therefore, our study provides evidence for the function of PLK1 in human ESCC and may shed a light of a novel strategy for cancer therapy.

Acknowledgements: This work was financially supported by the National Natural Science Foundation of China (30901779) and the Natural Science Foundation of Shandong Province, China (ZR2009CM019 and ZR2014HL058).

\section{References}

[1] PARKIN DM, BRAY FI, DEVESA SS. Cancer burden in the year 2000. The global picture. Eur J Cancer 2001; 37 Suppl 8: S4-66. doi: 10.1016/S0959-8049(01) 00267-2

[2] ENZINGER PC, MAYER RJ. Esophageal cancer. N Engl J Med 2003; 349: 2241-2252. doi: 10.1056/NEJMra035010 http:// dx.doi.org/10.1056/NEJMra035010

[3] BARR FA, SILLJE HH, NIGG EA. Polo-like kinase and the orchestration of cell division. Nat Rev Mol Cell Biol 2004; 5: 429-440. doi: 10.1038/nrm1401 http://dx.doi.org/10.1038/ $\underline{\text { nrm } 1401}$

[4] ZHAO C, GONG L, LI W, CHEN L. Overexpression of Plk1 promotes malignant progress in human esophageal squamous cell carcinoma. J Cancer Res Clin Oncol 2010; 136: 9-16. doi: 10.1007/s00432-009-0630-4 http://dx.doi.org/10.1007/ s00432-009-0630-4

[5] HAN DP, ZHU QL, CUI JT, WANG PX, QU S et al. Pololike kinase 1 is overexpressed in colorectal cancer and participates in the migration and invasion of colorectal cancer cells. Med Sci Monit 2012; 18: 237-246. doi: 10.12659/ MSM.882900

[6] FRISTRUP N, ULHØI BP, BIRKENKAMP-DEMTRODER K, MANSILLA F, SANCHEZ-CARBAYO M et al. Cathepsin E, maspin, $\mathrm{Plk}$, and survivin are promising prognostic protein markers for progression in non-muscle invasive bladder cancer. Am J Pathol 2012; 180: 1824-1834. doi: 10.1016/j.ajpath. 2012.01.023

[7] TAKAI N, MIYAZAKI T, FUJISAWA K, NASU K, HAMANAKA R et al. Expression of polo-like kinase in ovarian cancer is associated with histological grade and clinical stage. Cancer Lett 2001; 164: 41-49. doi: 10.1016/S0304 -3835(00)00703-5

[8] KNECHT R, ELEZ R, OECHLER M, SOLBACH C, VON ILBERG $\mathrm{C}$ et al. Prognostic significance of polo-like kinase (PLK) expression in squamous cell carcinomas of the head and neck. Cancer Res 1999; 59: 2794-2797.

[9] KNECHT R, OBERHAUSER C, STREBHARDT K. PLK (polo-like kinase), a new prognostic marker for oropharyngeal carcinomas. Int J Cancer 2000; 89: 535-536. doi: 10.1002/10970215(20001120)89: 6<535: : AID-IJC12>3.0.CO; 2-E http:// dx.doi.org/10.1002/1097-0215(20001120)89:6<535::AIDIJC12>3.0.CO;2-E

[10] SPANKUCH-SCHMITTB, BEREITER-HAHN J, KAUFMANN M, STREBHARDT K. Effect of RNA silencing of polo-like kinase-1(PLK1) on apoptosis and spindle formation in human cancer cells. J Natl Cancer Inst 2002; 94: 1863-1877. doi: $10.1093 /$ jnci/94.24.1863 http://dx.doi.org/10.1093/ jnci/94.24.1863 
[11] LIU X, ERIKSON RL. Polo-like kinase (Plk)1 depletion induces apoptosis in cancer cells. Proc Natl Acad Sci U S A 2003; 100: 5789-5794. doi: 10.1073/ pnas. 1031523100

[12] NOGAWA M, YUASA T, KIMURA S, TANAKA M, KURODA $\mathrm{J}$ et al. Intravesical administration of small interfering RNA targeting PLK-1 successfully prevents the growth of bladder cancer. J Clin Invest 2005; 115: 978-985. doi: 10.1172/ JCI200523043

[13] KAWATA E, ASHIHARA E, KIMURA S, TAKENAKA K, SATO K et al. Administration of PLK-1 small interfering RNA with atelocollagen prevents the growth of liver metastases of lung cancer. Mol Cancer Ther 2008; 7: 2904-2912. doi: 10.1158/1535-7163.MCT-08-0473. http://dx.doi. org/10.1158/1535-7163.MCT-08-0473

[14] YUE W, JIN YL, SHI GX, LIU Y, GAO Y et al. Suppression of $6 \mathrm{~A} 8$ alpha-mannosidase gene expression reduced the potentiality of growth and metastasis of human nasopharyngeal carcinoma. Int J Cancer 2004; 108: 189-195. DOI: 10.1002/ ijc.11536 http://dx.doi.org/10.1002/ijc.11536

[15] WEIDNER N, SEMPLE JP, WELCH WR, FOLKMAN J. Tumor angiogenesis and metastasis: correlation in invasive breast carcinoma. N Engl J Med 1991; 324: 1-8. doi: 10.1056/NEJM199101033240101 http://dx.doi.org/10.1056/ NEJM199101033240101

[16] MUKHERJEE P, SOTNIKOV AV, MANGIAN HJ, ZHOU JR, VISEK WJ et al. Energy intake and prostate tumor growth, angiogenesis, and vascular endothelial growth factor expression. J Natl Cancer Inst 1999; 91: 512-523. doi: 10.1093/ jnci/91.6.512

[17] MANDARD AM, HAINAUT P, HOLLSTEIN M. Genetic steps in the development of squamous cell carcinoma of the esophagus. Mutat Res 2000; 462: 335-342. doi: 10.1016/ S1383-5742(00)00019-3 http://dx.doi.org/10.1016/S13835742(00)00019-3

[18] IKEGUCHI M, OKA S, GOMYO Y, TSUJITANI S, MAETA $\mathrm{M}$ et al. Combined analysis of $\mathrm{p} 53$ and retinoblastoma protein expressions in esophageal cancer. Ann Thorac Surg 2000; 70: 913-917. doi: 10.1016/S0003-4975(00)01719-7 http://dx.doi. org/10.1016/S0003-4975(00)01719-7

[19] IKEGUCHI M, SAKATANI T, UETA T, KAIBARA N. Cyclin D1 expression and retinoblastoma gene protein ( $\mathrm{pRB}$ ) expression in esophageal squamous cell carcinoma. J Cancer Res Clin Oncol 2001; 127: 531-536. http://dx.doi.org/10.1007/s004320100265
[20] TONG T, ZHONG Y, KONG J, DONG L, SONG Y et al. Overexpression of Aurora-A contributes to malignant development of human esophageal squamous cell carcinoma. Clin Cancer Res 2004; 10: 7304-7310. doi: 10.1158/ 1078- 0432. CCR-04-0806

[21] WANG Q, LU J, YANG C, WANG X, CHENG L et al. CASK and its target gene Reelin were co-upregulated in human esophageal carcinoma. Cancer Lett 2002; 179: 71-77. doi: 10.1016/S0304-3835(01)00846-1 http://dx.doi.org/10.1016/ $\underline{\mathrm{S} 0304-3835(01) 00846-1}$

[22] ZHOU J, WANG H, LU A, HU G, LUO A et al. A novel gene, NMES1, downregulated in human esophageal squamous cell carcinoma. Int J Cancer 2002; 101: 311-316. doi: 10.1002/ ijc.10600 http://dx.doi.org/10.1002/ijc.10600

[23] TOKUMITSU Y, MORI M, TANAKA S, AKAZAWA K, NAKANO $S$ et al. Prognostic significance of polo-like kinase expression in esophageal carcinoma. Int J Oncol 1999; 15: 687-692. doi: 10.3892/ijo.15.4.687 http://dx.doi.org/10.3892/ ijo.15.4.687

[24] FENG YB, LIN DC, SHI ZZ, WANG XC, SHEN XM et al. Overexpression of PLK1 is associated with poor survival by inhibiting apoptosis via enhancement of survivin level in esophageal squamous cell carcinoma. Int J Cancer 2009; 124: 578-588. doi: 10.1002/ijc.23990 http://dx.doi.org/10.1002/ ijc. 23990

[25] KUBOTA Y, KANEKO K, KONISHI K, ITO H, YAMAMOTO $\mathrm{T}$ et al. The onset of angiogenesis in a multistep process of esophageal squamous cell carcinoma. Front Biosci 2009; 14: 3872-3878. http://dx.doi.org/10.2741/3495

[26] JAIN RK. Normalization of tumor vasculature: an emerging concept in antiangiogenic therapy. Science 2005; 307: 58-62. doi: 10.1126/science.1104819 http://dx.doi.org/10.1126/ science. 1104819

[27] KITADAI Y, ONOGAWA S, KUWAI T, MATSUMURA S, HAMADA $\mathrm{H}$ et al. Angiogenic switch occurs during the precancerous stage of human esophageal squamous cell carcinoma. Oncol Rep 2004; 11: 315-319. doi: 10.3892/ or.11.2.315

[28] LI Z, SHIMADA Y, UCHIDA S, MAEDA M, KAWABE A et al. TGF-alpha as well as VEGF, PD-ECGF and bFGF contribute to angiogenesis of esophageal squamous cell carcinoma. Int J Oncol 2000; 17: 453-460. doi: 10.3892/ ijo.17.3.453 